\title{
BP-SY-3-3
}

\section{Optoelectronic devices for smart healthcare applications}

\author{
Young Min SONG*
}

School of Electrical Engineering and Computer Science, Gwangju Institute of Science and Technology, Gwangju, Korea

Lecture: The diverse vision systems found in nature can provide interesting design inspiration for imaging devices, ranging from optical subcomponents to digital cameras, with more desirable optical characteristics compared to conventional imagers. The advantages of natural vision systems include high visual acuity, wide field of view, wavelength-free imaging, improved aberration correction and depth of field, and high motion sensitivity. Recent advances in soft materials, ultrathin electronics/optoelectronics have facilitated the realization of novel processes and device designs that mimic biological vision systems. This presentation highlights recent progress and continued efforts in the research and development of optoelectronic devices and imaging systems for smart healthcare applications. 